\title{
Design and Optimal Control of Line of Sight Stabilization of Moving Target
}

\author{
Ajeet Singh ${ }^{1}$, Ritula Thakur ${ }^{2}$, Dr. S. Chatterjee ${ }^{3}$ \\ M.E Student ${ }^{1}$, Assistant Professor ${ }^{2}$, Professor and Head ${ }^{3}$ \\ Department of Electrical Engineering, NITTTR, Chandigarh
}

\begin{abstract}
The Line-of-Sight stabilization and tracking control based on gyro stabilized platform is required to isolate Line of sight from the movement and vibration of carrier and ensure pointing and tracking for target in electro-optical tracking system. This work describes the design of a high performance controller for an electromechanical target tracking system with an optical sensor for sighting. The control law has been obtained using a linear model of the electro mechanical system. The modelling of the system has been carried out using the experimental frequency response data. The PID adesigning of control system methodology has been used to design the controller and study the comparative analysis using both controller and optimized tuned performance is also seeing by using the designing the PID controller in different loop analysis and study responses of system by using step response and frequency response analysis by using the bode diagram so as to stabilize the line of sight stabilization and tracking control system. The mixed sensitivity optimisation problem has been posed and weighting functions are selected by using the gradient and dissent method having more numbers of iterations so that they not only encapsulate all the design goals but also accommodate the modelling uncertainties. A stabilising controller with excellent robustness properties has been obtained. Investigator has used MATLAB for the designing of controller and simulated the system for a required criterion of the system.
\end{abstract}

Keywords: controller, stabilization, optimal, lag, lead

\section{Introduction}

The Line-of-Sight is the vector drawn between an imaging sensor and target. Electro-optical (EO) imaging sensors mounted on a mobile platform usually require some form of control to stabilize the sensor pointing vector along the target LOS[1][2][3]. Such a system is termed as LOS Stabilization System. It is an essential feature of electro- optical imaging tracking system on a movable carrier, such as infrared missile seeker, airborne electro-optical detector, etc. This technology is applied to isolate the LOS of sensor from carrier disturbance, in order to guarantee accurate aiming and tracking for the target in inertial space. LOS stabilization takes the rate gyro is a type of gyroscope, which rather than indicating direction, indicates the rate of change of angle with time[4]. Rate gyro is used to measure a rate of angular moment which is mounted on the stabilized axis as the speed sensor of inertial space to compose of the inertial platform. The control system manipulates the platform, which is driven directly by DC motor, and keeps LOS of imaging sensor stabilized. The stabilization system must limit the amount of image motion in the field of view of the optical sensor during a frame i.e. sensor integration period. The allowable jitter is a function of the sensor resolution and beam width of the pointing device, and these in turn are usually determined by the angular substance of the target at the desired operating range. The torque disturbances in the system can be due to bearing and motor friction, unbalanced aerodynamics, vibration forces from on-board mechanisms, spring torque forces from wires or flexures or due to some non-intuitive torques. In design of tracking system controller design is carried out for two-axis gimbals[5]. The LOS is stabilized in elevation as well as in azimuth with a high degree of disturbance in both the axes. High precision two-axis gimbals are engineered with very good stiffness properties. The inertial rate of the payload is sensed by very high performance dynamically tuned gyros (DTG), which are characterized by very low drift values of the order of $0.00001 \mathrm{deg}$. /sec. Two stabilization loops work simultaneously for the overall stabilization of the optical sensor in the space. The plant model is generated using experimental frequency response data. Design of control system for tracking of moving target is a significance of video surveillance. The system works on the principle of closed loop servo control[6]. A complete electro-optical tracking system consists of an imaging sensor (typically CCD), which is mounted on a two-axis stabilized servo platform, and a tracker, which controls the position of the platform, based on the scene observed through the imaging sensor[7]. In the case of a manual tracking system, an operator controls the position of the platform (via joystick, etc.) based on the image observed via a video monitor[8]. In this way, the operator will attempt to drive the platform so that the object of interest is fixed on the bore sight .In the case of an automatic tracking system, the operator is replaced by an electronic system which processes the video images directly in real-time to ascertain the position of a designated object with respect to the sensor bore sight[9][10]. This error is then used to control the platform such that the platform and attached sensors accurately follow the target. Line of sight stabilization is 
achieved by taking feedback from rate gyros mounted on azimuth and elevation gimbals of sight head. A twoaxis gimbaled stabilization system in air vehicles must stabilize the line of sight toward a target against the external motion induced by air vehicle manoeuvring and aerodynamic forces. It is well known that the target tracking and pointing performances of the air vehicles are largely affected by air vehicle motion decoupling capability. This introduces a servo drive model for a two-axis gimbaled stabilization system, and presents robust controllers for the air vehicle stabilization system[11]. The mutation results are compared to confirm the validity of the suggested simulation model and the control design procedures for the stabilization system applied to an air vehicle many kinds of air vehicles utilize two-axis gimbaled stabilization system consisting of target tracker, pitch/yaw gimbals and DC drive system[12]. The servo drive of such air vehicles is composed of tracking and search loops which commonly include the stabilization loop as an inner loop. The stabilization loop plays an important role in searching and tracking a target[13]. Generally, the two-axis gimbaled platform must achieve rapid and precise response characteristics, and also be stabilized during tracking a target. It is well known that tracking performance of air vehicles depend highly on stabilization performance which is able to isolate a target tracker from various disturbances induced by air vehicle body motion and vibration, even with satisfying the system stability margin and the desired high decoupling ratio between target tracker and air vehicle body. Here the gimbal pay load is having freedom of two degree so it is free to move in $\mathrm{X}$ and $\mathrm{Y}$ direction. The aim of control algorithm is to stabilize the pay load against the disturbances and same time it should follow the command given by the user[14]. A fire control system is an integral part of a weapon system of any military vehicle. The engagement capability of the modern weapon platforms has been increased over the conventional system by integration of new technologies. Observation / recognition range / capability have been increased under both static and dynamic conditions by stabilizing the line of sight (LOS). Aline of sight stabilization system consists of a precision electro-mechanical assembly, gimbals, optical sight and control electronics etc. This project report discusses and demonstrates the design of compensator/controller for tracking \& LOS stabilization by classical control methods using lag-lead networks. Figure 1 shows block diagram of control console servo platform arrangement and tracking of moving target access in the acquisition window[15]. A complete electro-optical tracking system consists of an imaging sensor (typically CCD), which is mounted on a two-axis servo platform, and a tracker, which controls the position of the platform, based on the scene observed through the imaging sensor. In the case of a manual tracking system, an operator controls the position of the platform (via a joystick, etc.) based on the image observed via a video monitor. In this way, the operator will attempt to drive the platform so that the object of interest is fixed on the bore sight. In the case of an automatic tracking system, the operator is replaced by an electronic system which processes the video images directly in real-time to ascertain the position of a designated object with respect to the sensor bore sight. The primary objective of the complete EO Tracking System is to control the position of the platform and attached sensor such that the object of interest viewed through the sensor appears at the centre of the field of view (Bore sight) and is maintained as close as possible to this point as the object moves[16]. Therefore, the control system used in an EO Tracking System requires an outer position loop which controls the platform based on the target position with respect to the Bore sight (Target to Bore sight Error).Figure 1 shows the block diagram of automatic video tracking system with stabilization[22].

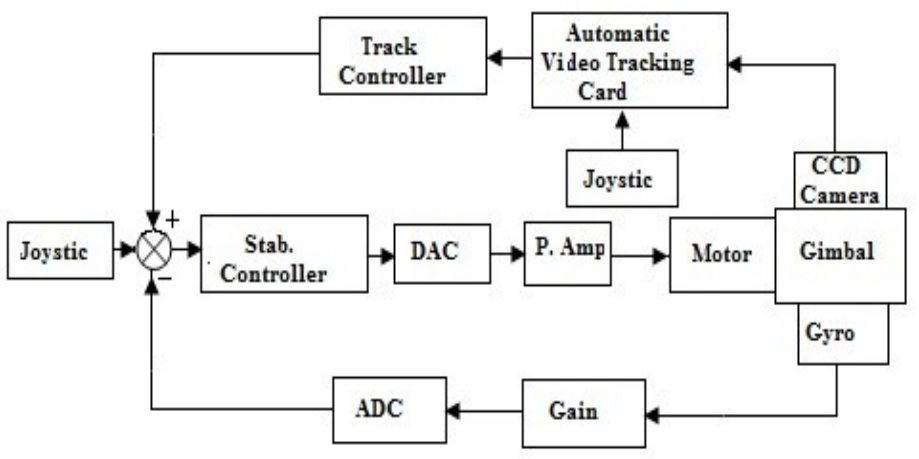

Fig.1.4 Bloc Diagram of Automatic Video Tracking with Stabilization

II. Simulink Model Development of Stabilization

Stability Robustness is required to take care of parameter variations and un-modeled uncertainties. For parameter variations, it is generally expressed in terms of gain margin $(\geq 4 \mathrm{~dB})$ and phase margin ( $\geq 30$ degree). Damping coefficient of 0.3 to 0.7 is considered for undershoot system. There are two considerations for the gain at low frequencies[21]. 
- If attenuation is $0.2 \mathrm{mil}$ and disturbance is $300 \mathrm{mils} / \mathrm{sec}$ so corresponding LOS position is $47.74 \mathrm{mil}$ if $1 \mathrm{~Hz}$ frequency of system hence required gain is $48 \mathrm{~dB}[16]$.

- If attenuation is $0.2 \mathrm{mil}$ and disturbance is $100 \mathrm{mils} / \mathrm{sec}$ so corresponding LOS position is $5.305 \mathrm{mil}$ if $3 \mathrm{~Hz}$ frequency of system hence required gain is $29 \mathrm{~dB}$.

The stabilization loop goal is given in table 4.2

Table 1 Stabilization loop parameter

\begin{tabular}{|l|l|}
\hline System parameter & Values \\
\hline Armature resistance of DC motor & $4.7 \Omega$ \\
\hline Armature inductance of DC motor & $0.0066 \mathrm{H}$ \\
\hline Electromotive constant & 1.54 \\
\hline Moment of inertia of gimbal & $1 \mathrm{Kg} \cdot \mathrm{m}^{2}$ \\
\hline
\end{tabular}

Design requirement of the stabilization controller for achieve the controller performance of stabilize the servomechanism of gimbaled payload platform of line of sight of the system given in table 4.2 having stabilization sampling frequency is $4000 \mathrm{~Hz}$. Design of controller to stabilize the line of sight block diagram shown in figure 2. for which design the PID controller[17].

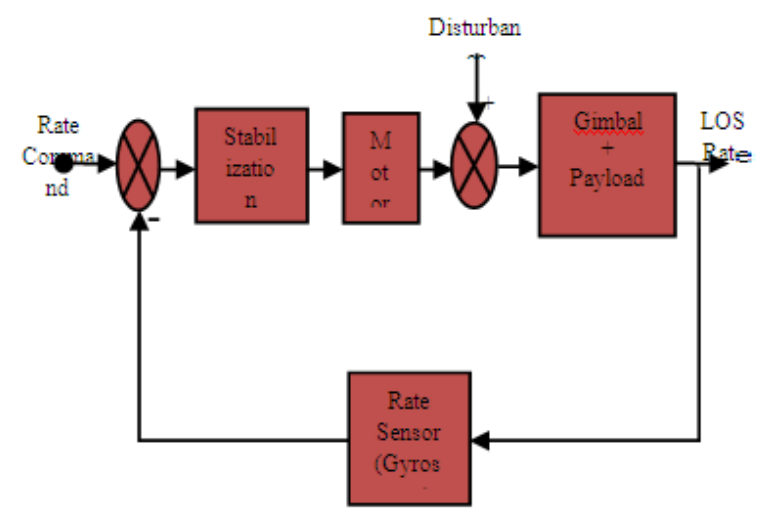

Fig.2 Block Diagram of Stabilization Loop

Transfer function of motor including gimbal payload is given by equation 4.1 by usin $T F=\frac{23333}{s^{2}+71526 s+259539}$ (1)

And rate sensor gyroscope having transfer function by equation (2) Gyroscope having damping ratio 0.7 natural frequency is $628.5 \mathrm{rad} / \mathrm{sec}$. and scale factor i.e. gain of gyroscope is $5.7 \mathrm{~V} / \mathrm{rad} / \mathrm{sec}[20]$.

$$
G_{\text {gyro }}=\frac{2250269803}{s^{2}+879.645 s+394784176}
$$

So the plant (motor and gimbal without resonance between motor and gimbal) and rate sensor is arranged in closed loop this loop is called as stabilization loop, the overall transfer function of the system is given by equation (3).

$$
T F_{\text {StabilizLoop }}=\frac{23333 s^{2}+2052475679 s+921149917861}{s^{4}+15949026 s^{3}+10265523376 s^{2}+28465540.4803 s+154967435.9826}
$$

The PID controller is used to stabilize the system and hence PID controller parameter Kps, Kis, and Kds which is tuned with the help of Zeigler-Nicholas tuning to fulfill the requirements of the system. The Simulink model of PID controller is shown in figure (5).

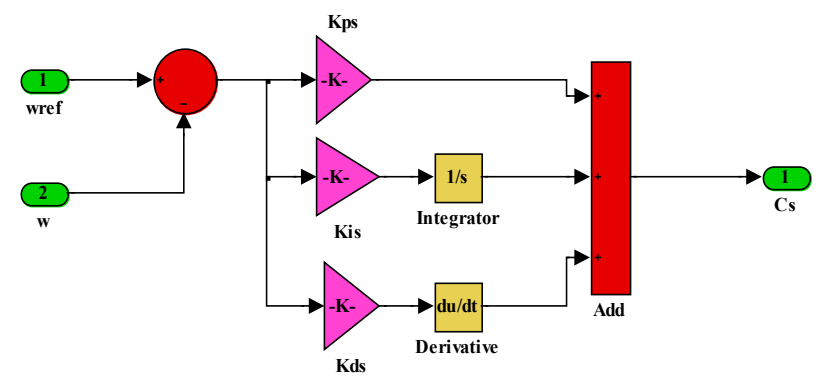

Fig.3 Simulink Model of PID Controller 
The Simulink model of stabilization of line of sight subsystem having gimbaled and gyro for LOS rate measurement of the system. The PID controller is used to tune the response of line of sight stabilization control system by the Zeigler-Nicholas tuning in this method we tune the gain of proportional, integral and derivative action of PID controller with hit and trail method to stabilize the system. The accepted response of the system is achieved by controller gain values are given in table 2 .

Table 2 Selected Parameters of PID Stabilization controller

\begin{tabular}{|l|l|}
\hline PID Controller Parameter & Gain \\
\hline Proportional Action Gain & $7.81663950931298 \mathrm{e}-09$ \\
\hline Integral Action Gain (Kis) & $4.33911275197983 \mathrm{e}-14$ \\
\hline Derivative Action Gain (Kds) & 0.000123246564101689 \\
\hline
\end{tabular}



Fig.4 Simulink of Line of Sight Stabilization System

\section{Results And Discussion}

Using the controller development in the previous sections the dynamic performance of line of sight stabilization and tracking controller operated system by using PID, Compensator and optimal controller and compare the responses of effectiveness of the controller in terms of controller parameters. Settling time, Rise time, Peak overshoot, Gain margin, Phase margin line of sight stabilization system controlled by PID controller shown in figure 6 . The result shows that the rise time settling time $0.0311 \mathrm{sec}$, rise time $0.0057 \mathrm{sec}$ and peak overshoot 22.8 percent[19]. The controller parameter values are $\mathrm{Kps}=0.00000000781$, $\mathrm{Kis}=0.00000000000004339$ and $\mathrm{Kds}=0.000123$. Achive bandwidth $\approx 0$ to $28.64 \mathrm{~Hz}$ by tuning the PID controller but used frequency band for the line of sight stabilization control appropriate $25 \mathrm{~Hz}$ to $50 \mathrm{~Hz}$ so we get very low bandwidth in the maximum range.

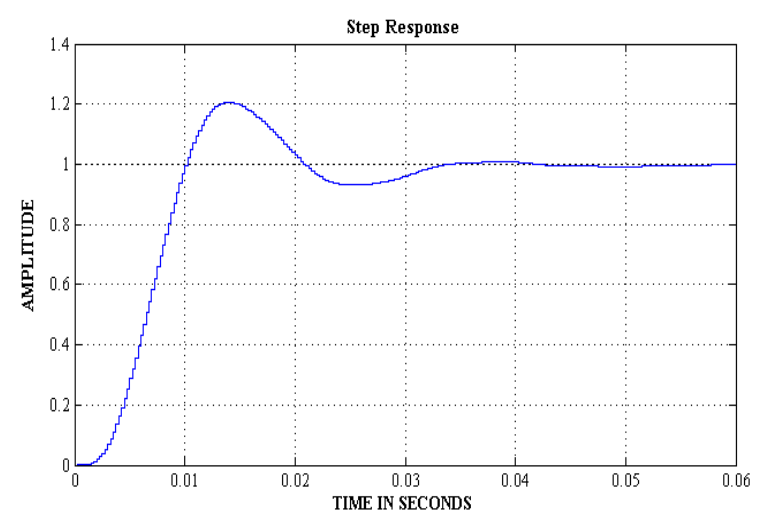

Fig. 5 Unit Step Response of Line of Sight Stabilization

Line of sight stabilization system controlled by PID controller bode response shown in figure 5.2 the result shows gain margin $3.52 \mathrm{~dB}$ and phase margin 29.2 . 




Fig.6 Bode Response of Line of Sight Stabilization

line of sight stabilization and tracking system controlled by PID controllers in the stabilization and tracking loop with positive feedback shown in figure 5.3. The result shows that the rise time settling time 0.11 seconds, rise time 0.025 seconds and peak overshoot 7.53 percent. The stabilization loop controller parameter values are $\mathrm{Kps}=2.10180179690939 \mathrm{e}-10$, Kis $=6.28833377115443 \mathrm{e}-15$ and $\mathrm{Kds}=.7 .83994856114218 \mathrm{e}-08$ with filter coefficient 0.0035 and tracking loop controller parameter $\mathrm{Kpt}=-2545008843229.17$, Kit = 1911220082395.12, and $\mathrm{Kdt}=-341602568351.156$ with filter coefficient 123.745. In the selection of the PID controller parameter by Ziegler-Nichols step response tuning to achieve the appropriate response. Line of sight stabilization and tracking control system controlled by two PID controller are used in the tracking and stabilization loop the bode response shown in figure 5.4 the result shows gain margin $40.2 \mathrm{~dB}$ and phase margin 132 degree having bandwidth in the range of

13.7 Hz. Table for PID Controller response

\begin{tabular}{|c|c|l|l|l|l|}
\hline $\begin{array}{c}\text { rise } \\
\text { Time }\end{array}$ & $\begin{array}{c}\text { Setting } \\
\text { Time }\end{array}$ & $\begin{array}{c}\text { Peak } \\
\text { Overshoot }\end{array}$ & $\begin{array}{c}\text { Peak } \\
\text { Time }\end{array}$ & $\begin{array}{c}\text { Gain } \\
\text { Margin }\end{array}$ & $\begin{array}{c}\text { Phase } \\
\text { Margin }\end{array}$ \\
\hline 0.025 & 0.11 & 7.53 & 0.056 & 40.2 & 132 \\
\hline
\end{tabular}

\section{Conclusion}

Non-linear behavior of line of sight stabilization and tracking control system introduces a challenge in low bandwidth faster response and negligible disturbances. In this real time control system application which is having large environmental disturbances resulting undesired echoes should be reduced. The present work gives an overview of PID controller. Besides these controllers work presents the optimization through gradient decent method. In this system two PID controller are used in the stabilization feedback and tracking loop respectively and the challenge in system is to tuning the both the stabilization and tracking controllers simultaneously. To achieve the response of tracking of moving target investigator investigate the control system parameter like rise time, settling time and percentage of peak overshoot. To overcome the limitation of PID and lead or lag controller has been designed. Simulation response controller shows that it reduces the settling time, rise time as compare to PID controller. To achieve the further improvement of PID controller the gradient decent optimization technique is used. The simulation results shows that it improve the step response of the system reduces the settling time and rise time of the system.

\section{References}

[1]. Michael K. Masten and Henry R. Sebesta, "Line-of-Sight Stabilization/Tracking Systems: An Overview", Proceeding of the IEEE Conference on American Control, Minneapolis, MN, USA, pp. 1477-1482,10-12 June 1987.

[2]. $\quad$ Peter J. Kennedyand Rhonda L. Kennedy, "Direct Versus Indirect Line of Sight (LOS) Stabilization", IEEE Transactions on Control Systems Technology, Vol. 11, and No.1, pp. 3-15 January 2003.

[3]. Vladimir N. Dobrokhodov, Isaac I. Kaminer, Kevin D. Jones, and Reza Ghabcheloo, "Vision-Based Tracking and Motion Estimation for Moving targets using Small UAVs", Proceedings of the IEEE Conference on American Control Minneapolis, Minnesota, USA, June 14-16, 2006.

[4]. Dr. H. Ambrose, Z. Qu and R. Johnson, "Nonlinear robust control for a passive line-of-sight stabilization system", Proceeding of the IEEE conference on Control Application, Mexico City, Mexico, pp. 942-947, September 5-7, 2001

[5]. Tian Qi, Wenzhou Su and Jie Chen, "Optimal Tracking Design and Performance Analysis for LTI Systems with Quantization Effects", Proceeding of the $48^{\text {th }}$ IEEE Conference on Decision and Control, China, pp.4945-4950, December 16-18, 2009.

[6]. Chong Jiang, Dexin Zou, Qingling Zhang, and Heli Hu, "Optimal Tracking Control for a Class of Large-Scale Interconnected system with Time-varying Delay", Proceeding of the International Conference IEEE on Control and Automation Guangzhou, China, pp.2529 - 2534, May 30 June 1, 2007.

[7]. Zhong-Hua Li and Miroslav Krstic, "Optimal Design of Adaptive Tracking Controllers for Nonlinear Systems", Proceedings of the American Control Albuquerque conference, New Mexico,Vol. 2, pp. 1191-1197, June 1997. 
[8]. Hongwei Zhang, Frank L. Lewis, and Zhihua Qu "Lyapunov, Adaptive, and Optimal Design Techniques for Cooperative Systems on Directed Communication Graphs", IEEE Transactions on Industrial Electronics, Vol. 59, pp. 3026-3041,7 July 2012.

[9]. Yandong Zhao and Xianli Chen, "Design of Optimal Tracking Controller for Systems with Control-Affine Form", Proceedings of the IEEE International Conference on Automation and Logistics, Jinan, China, pp.2472-2476, August 18 - 21, 2007.

[10]. Maria Nevia Ferrara and Andrea Torre, "Automatic moving targets detection using a rule-based system: comparison between different study cases", Proceeding of international symposium on Geosciences and remote sensing, Vol. 3, pp. 1593-1595, 6-10 July 1998.

[11]. Liang Sun and Hong-Wei GAO, “Approximately Optimal Tracking Controller Design for Nonlinear Interconnected Large-Scale Systems with Time Delays", Proceeding of the $7^{\text {th }}$ World Congress on Intelligent Control and Automation, Chongqing, China, pp. 905-910,25 - 27 June 2008.

[12]. Cheng-Ming Zhang, Gong-You Tang and Shi-Yuan Han, "Approximate design of optimal tracking controller for systems with delayed state and control", Proceedings of the International Conference on Control and Automation Christchurch, New Zealand, pp. 1168-1172, 9-11 December 2009.

[13]. Ying-Lu Zhang and Gong-You Tang, "Approximate Design of Optimal Tracking Controller for Linear Systems with Time-delay", Proceedings of the International Conference on Systems, Man, and Cybernetics, Taipei, Taiwan,pp.4557-4562, 8-11 October 2006.

[14]. Xiao-Han Wang, Gong-You Tang, and Hai-Hong Wang, "Approximate Design of Optimal Output Tracking Controller for TimeDelay Systems with Sinusoidal Disturbances", Proceedings of the $9^{\text {th }}$ International Conference on Control, Automation, Robotics and Vision, Singapore, pp. 1-6, 5-8 December 2006.

[15]. Alan J. Lipton, Hironobu Fujiyoshi and Raju S. Patil, "Moving Target Classification and Tracking from Real-time Video", Proceedings of the IEEE $4^{\text {th }}$ Workshop on Applications of Computer Vision, Princeton, pp.8-14, 19-21 October 1998.

[16]. Peter I. Corke and Seth A. Hutchinson, "Real-Time Vision, Tracking and Control", Proceedings of the International Conference on Robotics \& Automation, San Francisco, CA, pp.622-629, 24-28 April 2000.

[17]. Ismail Haritaoglu, David Harwood and Larry S. Davis, "W4: Real-Time Surveillance of People and Their Activities", IEEE Transactions on pattern analysis and machine intelligence, Vol. 22, No.8, pp. 809-830, Aug 2000. 